\title{
Study on the postural rehabilitation devices used in the prevention of postural defects and the need to use a smart orthosis
}

\section{LUCA Cătălina ${ }^{1}$, FUIOR Robert ${ }^{1}$, CORCIOVĂ Călin ${ }^{1}$}

Corresponding author: LUCA Cătălina, E-mail: luca.katalina@yahoo.com

1. Faculty of Medical Bioengineering, "University of Medicine and Pharmacy Grigore T Popa", Iasi, Romania

\begin{abstract}
Introduction. Postural defects are a real challenge for the contemporary world. Scoliosis, kyphosis and lordosis are diseases of the spine, with various evolutions and multiple changes of the other components of the thorax (shoulders, shoulder blades, ribs), as well as of the intrathoracic and even abdominal organs. Material and method. This paper presents a study on the need to use a smart orthosis in the prevention of postural defects, a study that is the preamble to the physical realization of such an orthosis. The smart orthosis will use inertial sensors capable of calculating orientation angles in a portable monitoring system that will calculate and represent the curvature of the spine. It is hoped that finding a non-invasive solution to identify the shape of the human back can help reduce the time required for medical rehabilitation sessions or can prevent possible postural defects. Results and discussions. In this study, the existing orthoses on the market that could fall into the category of rehabilitation devices used in the prevention of postural defects were evaluated and an intelligent orthosis was designed. Conclusions. At this time, the existence of an intelligent orthosis in the prevention of postural defects would lead to an improvement in health among the population. Also, this prosthesis would crown the work of physiotherapists by maintaining the results obtained.
\end{abstract}

Keywords: postural defects, smart orthosis, prevention, rehabilitation, health improvement,

\section{Introduction}

A number of musculoskeletal disorders of the spine are closely related to vicious positions, including structural deformity of the spine and back pain $(1-5)$. Spinal disorders can occur in all categories of the population, and are largely due to lifestyle, excessive population growth in certain areas of the globe, and an aging population worldwide. The correct attitude of the body is a sign of physical and mental balance, a result of the normal and harmonious development of the body. Scoliosis, kyphosis and lordosis are diseases of the spine, with various evolutions and multiple changes that influence the functioning of the body and internal organs. Defective posture is most common among adolescents and has been reported as the most important risk factor for neck, back and shoulder pain. If a posture is maintained for a long time, cumulative effects may begin to occur. The results of Cho's study showed a high rate of posture characterized by the neck being bent forward $(25 \%)$, associated with a high percentage of adolescents (43\%) who spend long periods of time in front of the TV and computer. Computer use among children aged 11 to 15 years has been associated with poor posture in other studies; these works also mention physical inactivity, depression and stress as causes among children (6). Age has been reported as a risk factor for increased back pain, which occurs especially after the age of 12 . At the national level, poor posture is a major health problem that occurs in school-age children and can seriously affect the health of the future adult (7). In figure 1 you have the image of a four years child with dextroconvex $\mathrm{S}$ scoliosis.

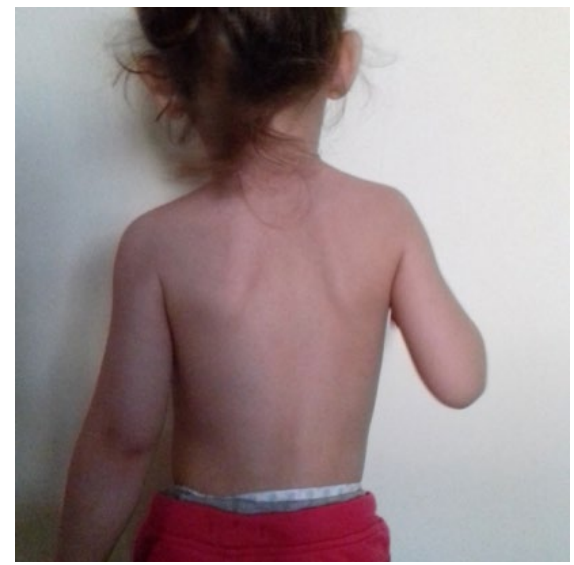

Fig. 1. Patient with dextroconvex S scoliosis

The concept of multidisciplinary rehabilitation of these spinal disorders includes surgery, medical and orthotics and back muscle strengthening exercises to counteract postural deviations of patients. For non-surgical and nonmedical interventions, conventional orthosis interventions must apply passive forces to the human body with orthosis to support the alignments of the trunk and control the deformities of the spine. Currently, rigid thoraco-lumbo-sacral orthoses (TLSO) are used in patients with progressive idiopathic scoliosis and soft lumbar corsets are used in patients with low back pain and the elderly with osteoporotic vertebral fracture (810). 


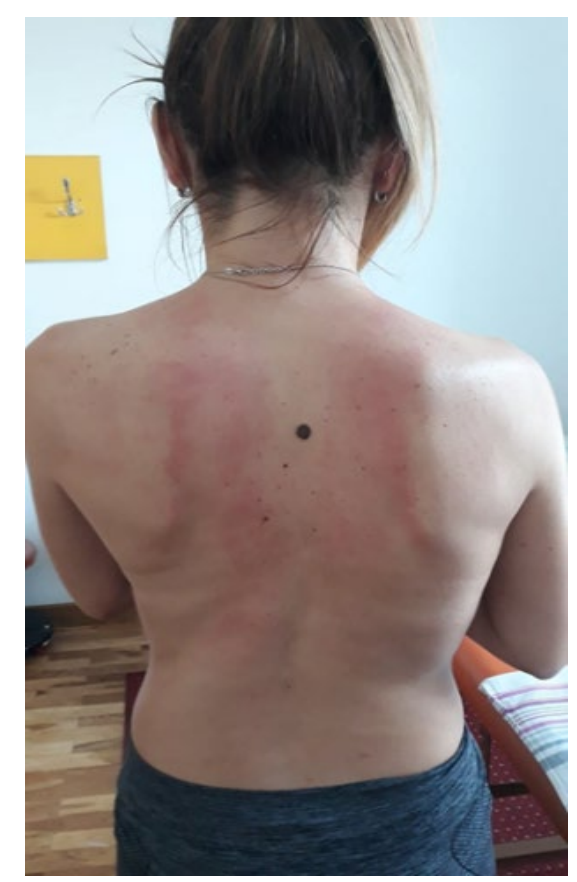

Fig. 2. Dextroconvex dorsal scoliosis and sinistroconvex lumbar scoliosis, with accentuated right costal relief, a slight clogging of the left thorax and asymmetry of the shoulder blades

To keep the torso upright with active muscle forces it is necessary to perform strengthening exercises for the back muscles. In most cases, patients do not perform the rehabilitation program suggested by the physiotherapist alone at home because patients are not motivated (11-12). A vicious daily posture in patients with spinal disorders can further damage the spine and regional pain due to its deformities. Poor posture is defined as any prolonged deviation from the "neutral spine". For postural correction, postural training was proposed and the school of the back appeared. In this rehabilitation program the patient is taught to use their own back muscles to keep the spine in natural curvature. Attempts are made to make the patient aware of his vicious posture in order to prevent the appropriate symptoms (13).

Postural training can facilitate the refinement of proprioceptive awareness of vertical posture and prevent the deterioration of spinal deformities, such as scoliosis and lordosis.

But using a smart system that would generate a "reminder" for maintaining a good posture could be an excellent prophylaxis for at-risk patients. A postural training device can help facilitate this therapeutic approach by providing continuous posture monitoring and feedback signals to patients when a "weak" posture is detected.

At this time, global trends are urging the population to engage in systematic physical activity to improve their health. Regular physical exertion has also been shown to influence longevity and quality of life. Exercise also strengthens the body from an immune point of view. An analysis of the locomotor system shows that physical activity improves skeletal mineralization and bone growth in children and adolescents, strengthens and stabilizes joints, strengthens tendon and ligament attachment, increases cross section and volume. Muscle increases tension, elasticity and muscle strength.

Physical activity also contributes to increasing lung capacity and thus to increasing the depth of respiration, improving the condition of the respiratory system. The nervous system during exercise undergoes changes by stimulating the process of maturation of the motor areas of the brain that contribute to the development of locomotor skills by improving the conduction of nerve impulses and locomotor coordination (14).

All the factors mentioned above prevent postural defects and play an important role in correcting postural defects. But if the patient is not stimulated he will not do the necessary physical exercises to maintain a correct posture.

\section{Matherial and Methods}

Postural defects can be divided into two categories: static defect and dynamic defect. Static defects are usually multiple and can be observed when the patient is at rest, sitting or standing. Defects of the legs and ankles, such as flat feet and valgus ankles, can be responsible for an obvious change in posture and can cause secondary defects such as hitting the knees and changing the curves of the spine. In these situations, patients have daily regional pain.

Dynamic posture is how the curvature of the spine changes when the patient moves, runs, or bends to lift something. In this situation the muscles and noncontractile structures must work to adapt to changing circumstances.

In order to maintain a correct posture, an intelligent orthosis must be designed to evaluate the patient's posture in both statics and dynamics.

\section{Types of smart orthoses on the market}

A posture-correcting orthosis should be light and comfortable to wear when the spine is in its neutral position. If the patient changes position by bending or turning the shoulders, the device should provide an easy correction.

One through device that I studied is the Marakym Posture Corrector is made of a light neoprene. It can be worn under or over clothing, and velcro straps make it easy to adjust for many body types and sizes. The manufacturers recommend specialized help for the first adjustment of this orthosis, which means that it is not a device that can be easily adapted to the patient's needs, but through certain adjustments (15).

Another device studied is a traditional posture corrector called Evoke Pro Back Posture Corrector. The Evoke Pro posture corrector has a modified 8-shape design, which 
achieves a better pressure distribution between the shoulder blades. Velcro straps allow you to adjust the tension and position the strap. The breathable, tight fabric fits on the back, and the padded straps wrap under the arms, without restricting movement (16).

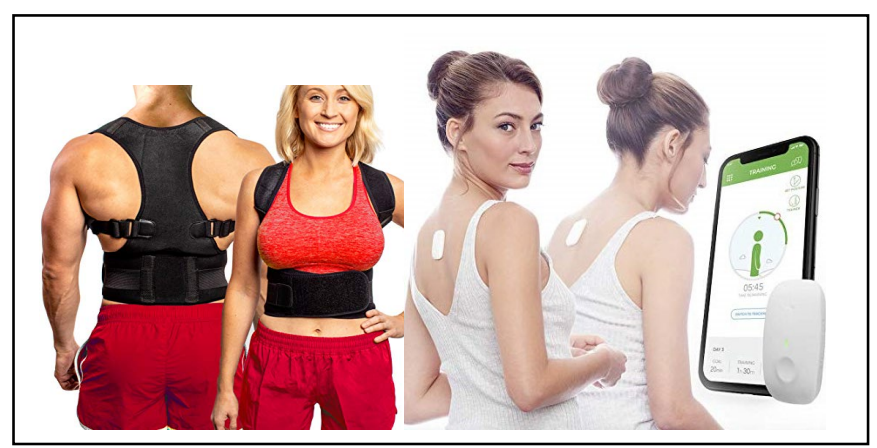

Fig. 3. Flexguard and Upright GO Posture Trainer images

Back pain can be reduced according to Flexguard manufacturers by ensuring better shoulder stability. This design is partly back (figure 3), partly posture corrector and all about the correct alignment of the spine. Flexguard Support Back Brace Posture Corrector is a handy option for people suffering from back pain due to a vicious posture (17).

There are also devices specially designed for those who carry out daily office activities. Upright GO Posture Trainer (figure 3) is a portable device that sticks to the center of the upper back with secure skin adhesives. When the patient's posture is no longer correct, the device vibrates to remind the patient to maintain a correct posture (18).

Another device has been specially designed to combat back pain. Better Back consists of unique straps that wrap around the patient's knees and turn any chair into an ergonomic chair. In this way it helps the patient to improve his posture and reduces pain. The manufacturers recommend wearing it for only 15 minutes a day and can help the patient train in a perfect posture (19).

\subsection{Methods used in the design of smart orthosis}

From the studies performed, it seems that at this moment there is no orthosis device designed to be designed for the entire spinal cord. Existing devices have some disadvantages such as the fact that they follow only the longitudinal and horizontal circumferences of the trunk and cannot provide any information directly related to the curves of the spine (20).

The system designed by us is designed to be used to monitor spinal posture in terms of instantaneous spinal length, continuously and in real time. In this way this device is intended to provide feedback signals to patients to correct their vicious posture.

Tensions that can occur in the spine due to a vicious posture can cause discomfort and pressure changes in the gluteal slit and in the groin area.
The medical device that should be developed should be a portable, easy-to-use and more innovative postural training system. For a complete postural correction this device should be able to measure the parameters related to the curvature of the spine. It should also be easy to wear and use so that it can provide postural information of daily activity and improve the feasibility and effectiveness of postural training.

The development of position sensors has undergone a huge increase in the last 10 years because we are talking about sensors that are studied and used in various fields such as human robotic applications, industrial, aerospace and biomedical applications. The technologies for making electronic circuits have advanced a lot and these sensors have become smaller in size and better in performance. Accelerometers and gyroscopes are commonly used to provide information on position and orientation in the aerospace and robotics industry.

For the analysis of the movements of the trunk, it is not enough to use these categories of sensors because it does not provide complete information about the curvature of the spine. These sensors can also be used to measure the kinematic parameters of body segments. Therefore, a portable and portable posture monitoring system cannot be built with these miniature sensors at the base, because studies have shown that they cannot perform full and real-time monitoring.

In the orthosis designed in our laboratory, for a complete monitoring of the vertebral curvature, we used inertial sensors and Flex sensors ass you can see in figure 4. By permanently determining the variation of the curves of the spine, we can also provide real-time feedback to the patient.

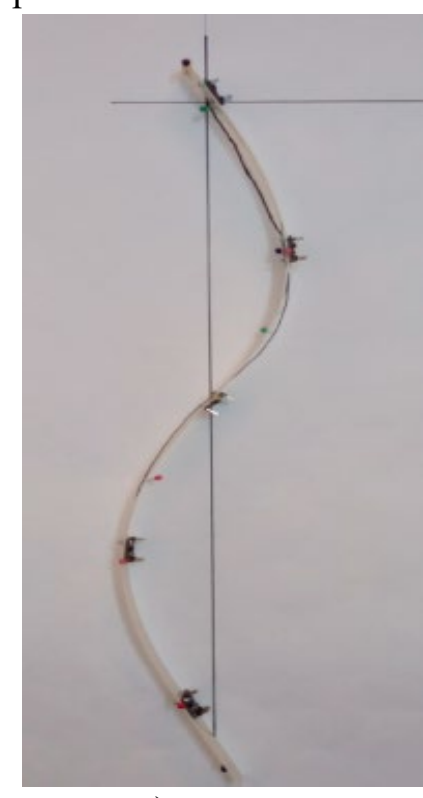

a)

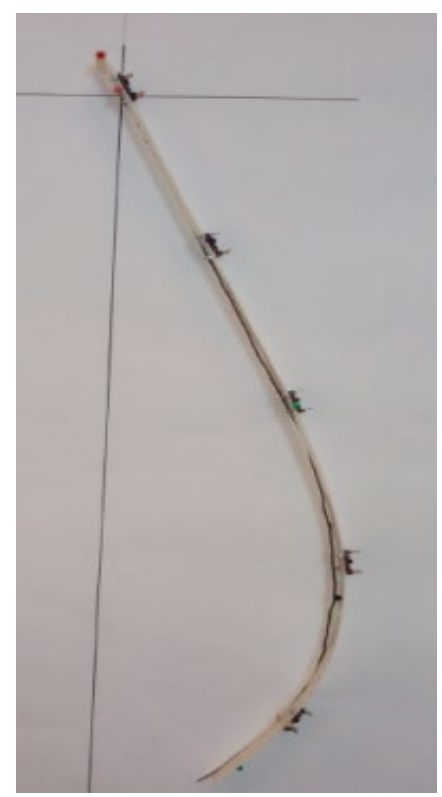

b)
Fig. 4. The scheme of placement possibilities in a) double max points positive curve and b) single max points positive curve 
The block diagram of the smart orthosis proposed by us includes Flex sensors, Inertial sensors, a GPS/GSM module, Bluetooth module that are all controlled by an Arduino Mega 2560- Central Unit as can be seen in figure 5 .

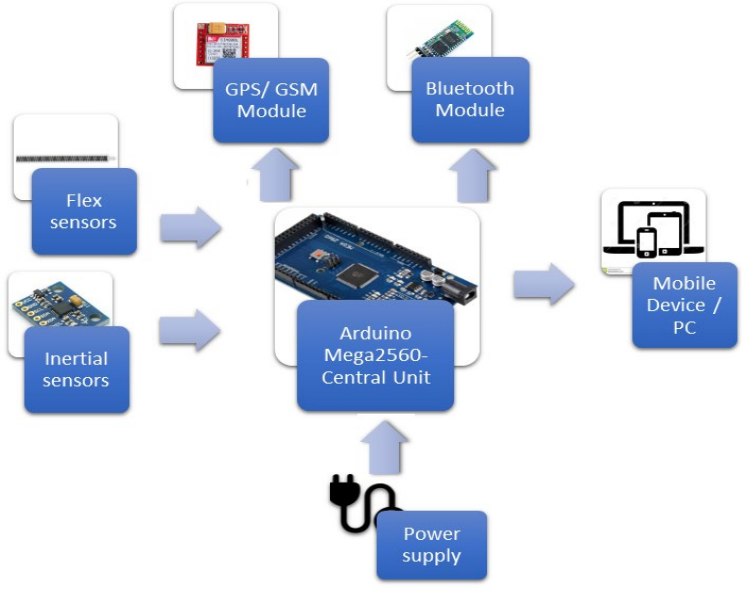

Fig. 5. Block diagram of the concept proposed by the intelligent orthosis

Our intention is to provide continuous feedback to both patients and physicians or treating physiotherapists. Therefore, all recorded data on changes in spine curvature are sent in real time to the patient via a mobile application. This mobile application is a permanent feedback of the orthosis so that the patient can selfcorrect his vicious positions. Also, our intention is to make an analysis of the signals collected in 24 hours and this analysis should be sent to the attending physician / physiotherapist in order to interpret the patient's evolution.

The features we want to implement in creating this smart orthosis used as a posture corrector include wearing comfort, ease of use and size reduction.

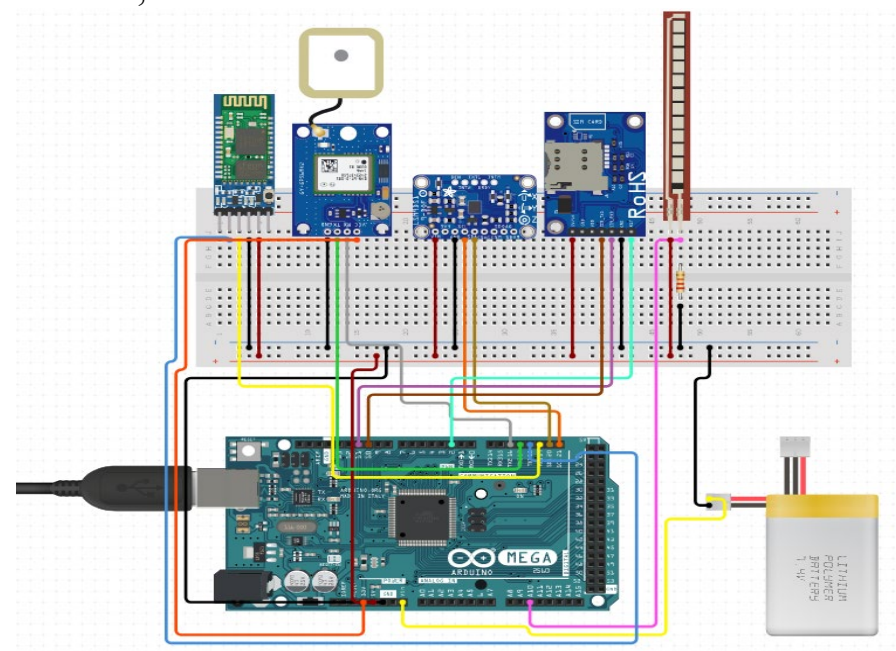

Fig. 6. Wiring diagram of the designed device

\section{Conclusions}

The purpose of this study is to design an intelligent orthosis that can detect postural changes in the variation of the curvature of the spine. This orthosis is intended to be used in the prevention of postural injuries and in guiding patients to maintain a correct posture of the spine during treatment during daily activities.

The smart orthosis was designed to be a portable and easy-to-use spine monitoring system that could be attached to the body to track changes in vertebral angles in daily activities. This orthosis could be used to collect information about the posture of the spine and the daily postural habits of users. In this way the device can provide real-time and continuous feedback to patients to correct vicious postures.

The likelihood of a patient regularly wearing a spinal corrector that is comfortable is higher than in the case of potentially robust devices. Another aspect taken into account by us is the design of the device and the way it interacts with the patient, specially designed so that the patient does not require technical or medical knowledge in use. Most posture correcting devices have universal dimensions. Our idea in fixing this device is based on adjustable straps combined with elastic bands that adapt to each patient.

\section{Author contributions.}

All the authors had the same contribution.

\section{Acknowledgements.}

This article belongs to the project ESTABLISHMENT OF RESEARCH DEPARTMENT INNOVATIVE METHODS OF TREATMENT OF NEUROLOCOMOTOR SYSTEM DISEASES,

Priority Axis no.1, 111-POC, SMIS- 121822.

\section{References:}

1. Ali RM, Green DW, Patel TC: Scheuermann's kyphosis. Current opinion in pediatrics 1999, 11(1):70-75.

2. Dworkin B, Miller NE, Dworkin S, Birbaumer N, Brines ML, Jonas S, Schwentker EP, Graham JJ: Behavioral method for the treatment of idiopathic scoliosis. Proceedings of the National Academy of Sciences of the United States of America 1985, 82(8):2493-2497.

3. Kratenova J, Zejglicova K, Maly M, Filipova V: Prevalence and risk factors of poor posture in school children in the Czech Republic. The Journal of school health 2007, 77(3):131-137.

4. Hignett S: Postural analysis of nursing work. Applied ergonomics 1996, 27(3):171-176. 
5. Womersley L, May S: Sitting posture of subjects with postural backache. J Manipulative Physiol Ther 2006, 29(3):213-218.

6. Wai Yin Wong* and Man Sang Wong, Smart garment for trunk posture monitoring: A preliminary study Scoliosis 2008, 3:7 doi:10.1186/1748-7161-3$7 \mathrm{http}: / / \mathrm{www}$.scoliosisjournal.com/content/3/1/7

7. WORLD REPORT ON DISABILITY, World Health Organization 2011, WHO Press, World Health Organization, 20 Avenue Appia, 1211 Geneva 27, Switzerland (pp 58-60)

8. Calmels P, Fayolle-Minon I: An update on orthotic devices for the lumbar spine based on a review of the literature. Revue du rhumatisme (English ed 1996, 63(4):285-291.

9. Levine AM: Spinal orthoses. American family physician 1984, 29(3):277-280.

10.Pfeifer M, Begerow B, Minne HW: Effects of a new spinal orthosis on posture, trunk strength, and quality of life in women with postmenopausal osteoporosis: a randomized trial. Am J Phys Med Rehabil 2004, 83(3):177-186

11.Nelson DA, Bouxsein ML: Exercise maintains bone mass, but do people maintain exercise? J Bone Miner Res 2001, (2):202-205.

12.Sinaki M, Wahner HW, Bergstralh EJ, Hodgson SF, Offord KP, Squires RW, Swee RG, Kao PC: Threeyear controlled, randomized trial of the effect of dose-specified loading and strengthening exercises on bone mineral density of spine and femur in nonathletic, physically active women. Bone 1996, 19(3):233-244.

13.Birbaumer N, Flor H, Cevey B, Dworkin B, Miller NE: Behavioral treatment of scoliosis and kyphosis. Journal of psychosomatic research 1994, 38(6):623628.

14. Marta Motow-Czyż1ABDE, Aneta Orczyk2ABDE, Marek Orczyk3AB Postural defects correction in the process of physical education and sport Physical Activity Review vol. 2, 2014 Research Methodology 15.https://marakym.com/

16.https://www.evokepro.com/evoke-pro-a300-posturecorrector/

17.https://www.geartekonline.com/product/wearables/b ack-posture-corrector/flexguard-back-posturecorrector/

18.https://www.uprightpose.com/en-gb/

19.https://getbetterback.com/.

20.Rață Bogdan-C., Rață Marinela, Antohe Bogdan, Morpho-functional implications of myofascial stretching applied to muscle chains: A case study Journal of Back and Musculoskeletal Rehabilitation, Volum: Vol. 31, Nr: issue 4, An: 2018, 749-758, ISSN: print 1053-8127, online 1878-6324, $\mathrm{IF}=0,982(2017), \quad$ DOI: $10.3233 / \mathrm{BMR}-170998$, WOS:000445002100020 